\title{
Open Access international - lokale Systeme, kooperative Netzwerke und visionäre Infrastrukturen
}

\author{
Wolfram Horstmann \\ Niedersächsische Staats- und \\ Universitätsbibliothek Göttingen \\ whorstmann@sub.uni-goettingen.de
}

Ein großer Bereich von Open-Access-Aktivitäten setzt beim nachhaltigen Betriebskonzept auf lokal verteilte, offene digitale Inhaltsspeicher in Bibliotheken und anderen Infrastruktureinrichtungen einer Institution. Inhalte werden also lokal vorgehalten - Wissenschaftlern soll aber selbstverständlich ein global umfassender Zugang auf Inhalte gewährt werden. Suchmaschinen wie OAISTER, BASE oder auch Google Scholar ermöglichen dies derzeit in einem ersten Schritt. Um aber das Potenzial eines verteilten, kooperativen Inhaltsmanagements tatsächlich auszuschöpfen, und eine homogene Dateninfrastruktur zu etablieren, muss die lokale Datenhaltung hohen Qualitätsstandards genügen. Besonders in Europa arbeiten lokale Systeme daher in regionalen, nationalen oder internationalen Netzwerken zusammen, etwa DINI in Deutschland oder DRIVER in Europa. Eine homogene Dateninfrastruktur, die Inhalte im offenen Zugriff bereitstellt, ist die Voraussetzung dafür, dass über das Internet Mehrwertfunktionen für Wissenschaftler, etwa Annotation und Begutachtung oder Bearbeitung und Publikation aufgebaut werden können.

Within the framework of sustainable operational planning, a large portion of open access activities depends upon decentralized open digital storage sites in libraries and other types of institutional infrastructures. While the content is maintained locally, scientists should, of course, be provided with a global, comprehensive content access. Search engines such as OAISTER, BASE or Google Scholar represent at present the first steps in this direction. However, in order to make the most of the potential benefits of a decentral, cooperative content management, and to establish a homogenous infrastructure, local data storage will need to meet high quality standards. Hence, especially in Europe one finds local systems working cooperatively in regional, national and international networks, as for example DINI in Germany or the European-wide DRIVER. A homogenous data infrastructure which offers open access is the prerequisite for developing surplus functions for scientists via Internet, such as annotations, peer review or revision and publication.

\section{Offene Digitale Archive}

Das Rückgrat der Open-Access (OA)-Aktivitäten wird von offenen digitalen Inhaltsspeichern (engl. Institutional Repositories, IRs) ${ }^{1}$ gebildet. Dies liegt nicht etwa daran, dass IRs auf der Linie der lautstärksten Verfechter von OA liegen, dem so genannten „Grünen Weg' ${ }^{62}$. Dies liegt auch nicht daran, dass OA im Bereich der elektronischen Zeitschriften, dem so genannten "Goldenen Weg“ aussichtslos wäre ${ }^{3}$. Der Grund dafür, dass man von einem Rückgrat sprechen kann, ist vielmehr, dass es nur im Bereich der IRs gelungen ist, tatsächlich systematische Fortschritte bei der infrastrukturellen Verankerung von OA zu machen. Und dies gilt insbesondere für Europa. Denn hier arbeiten Bibliotheken und zentrale Einrichtungen an Hochschulen und Forschungseinrichtungen auffällig intensiv und kooperativ an einer systematischen Vernetzung von IRs. Diese kooperative Einstellung ist bemerkenswert in einer sonst so fragmentierten Landschaft der wissenschaftlichen Informationsdienstleistung.

Und diese Kooperation ist besonders wichtig für die Zukunftsfähigkeit der OA-Idee, da vor allem wissenschaftliche Bibliotheken mit ihrem alt hergebrachten Mandat für die wissenschaftliche Informationsversorgung es dauerhaft leisten können, IRs und darauf aufbauende Dienste anzubieten, weiter zu entwickeln und als einen Standard in der Informationsversorgung zu etablieren. Anders als wissenschaftliche Abteilungen, deren Alltag durch ständig wechselnde Forschungsprojekte geprägt ist - und 
eben nicht durch die Bereitstellung nachhaltiger Dienstleistungen - haben Bibliotheken alle Möglichkeiten, wenn nicht sogar die Pflicht, dazu beizutragen Innovationen aus der wissenschaftlichen Kommunikation in den Regelbetrieb zu überführen. Es ist deshalb auch wohl nicht verwunderlich, dass selbst disziplinäre IRs wie das überaus erfolgreiche $\operatorname{arXiv}^{4}$ im technisch-physikalischen Sektor oder PubMedCentral ${ }^{5}$ im biomedizinischen Sektor von Bibliotheken betrieben werden.

\section{Lokale Systeme und regionale Vernetzung}

Betrachtet man die Landschaft Europäischer IRs, fällt zunächst die sehr hohe Anzahl von einzelnen IRs im internationalen Vergleich auf ${ }^{6}$. Einzelne IRs sind entscheidend für die Ablage lokaler Information, sind jedoch für Wissenschaftler bei der Informationssuche nur bedingt attraktiv, da sie lediglich jeweils den lokalen Ausschnitt der verfügbaren Information beinhalten. Wissenschaftler sind es aber gewohnt, bei der Informationssuche auf nationale oder internationale Datenbestände zuzugreifen und möchten nicht einen lokalen Katalog nach dem nächsten ,durchblättern'. Die kooperative Vernetzung von IRs ist daher auch ein Muss. Diese findet regional, national, kontinental oder global statt, und kann dabei technische oder thematische Ausprägungen annehmen. Die erste Stufe von Vernetzung ist auf regionaler Ebene zu beobachten, etwa in England im London-Leap-Consortium ${ }^{7}$ oder der WhiteRose-Partnership ${ }^{8}$. Dazu gibt es technisch motivierte Netzwerke, die dieselbe Plattform benutzen, etwa das DIVA-Netzwerk ${ }^{9}$ in Schweden. Die Motivation für diese Netzwerke ist jedoch weniger die Erstellung umfassender Inhaltssammlungen, sondern mehr die Zusammenarbeit beim Betrieb der

Horstmann: Open Access international - lokale Systeme, kooperative Netzwerke und S. 231 visionäre Infrastrukturen. ZfBB 54 (2007) 4-5, S.230-233.

IRs, etwa durch gemeinsame Nutzung von Ressourcen oder Plattformen.

\section{Nationale Netzwerke}

Auf der nächsten Stufe, der nationalen Vernetzung, sind wohl die stärksten Entwicklungen zu beobachten. In Deutschland ist es der Deutschen Initiative für Netzwerkinformation, DINI e.V. ${ }^{10}$ in international einzigartiger Weise gelungen, Qualitätsstandards für IRs zu etablieren. In einem Zertifikationsprozess können sich IRs einer Begutachtung unterziehen, die anhand eines Kriterienkataloges ein Zertifikat erteilt, das in seiner Breite von technischen, rechtlichen und organisatorischen Rahmenbedingungen wohl weltweit die höchste systematische ,Auszeichnung' für IRs darstellt. Bisher haben 19 IRs in Deutschland dieses Zertifikat erhalten, das gewährleistet, dass eine Standardisierung gegeben und damit eine zuverlässige Vernetzung möglich ist.

Die Niederlande verfolgen einen anderen Ansatz, in dem Betreiber von IRs nicht selbst auf eine vernetzende Organisation zugehen, sondern von einer Organisation, in diesem Falle SURF ${ }^{11}$, vernetzt werden. Das so entstandene DAREnet ${ }^{12}$, an dem rund 20 niederländische Hochschulen beteiligt sind, gehorcht technischen Richtlinien und stellt einen nationalen Zugriff auf alle in den lokalen IRs vorgehaltenen Inhalte sicher. Darüber hinaus konnten auf Basis dieses grundlegenden Netzwerkes spezialisierte Netzwerke aufgebaut werden. Für „Cream of Science“ beispielsweise wurden Hochschulen aufgefordert, die 10 ,besten' Wissenschaftler ihrer Institution zu nennen, deren Publikationen dann jeweils auf dem lokalen IR gesammelt wurden. Diese Wettbewerbssituation führte dazu, dass eine große Menge hochklassiger Publikationen erschlossen werden konnte und nun im OA verfügbar sind. Aber auch andere spezialisierte Netzwerke, wie eines für Abschlussarbeiten („Promise of Science“) konnten auf Basis von DAREnet aufgebaut werden.

In Großbritannien ist SHERPA ${ }^{13}$ die maßgebliche Organisation. Hier liegt der Schwerpunkt der Vernetzungsarbeit bei der politischen Unterstützung der OA-Idee für den Aufbau von IRs und bei der Etablierung weltweit verwendeter Spezialdienste. Mit RoMEO ${ }^{14}$ können rechtliche Rahmenbedingungen für die offene Anbietung von Verlagspublikationen abgefragt werden, mit OpenDOAR ${ }^{15}$ wird eine qualitätsgesicherte Registratur von IRs angeboten.

Frankreich bildet ein alternatives Modell von Vernetzung, bei dem lokale Publikationen national zentralisiert auf einer Plattform namens $\mathrm{HAL}^{16}$ abgelegt werden, das einen steilen Anstieg der Inhaltszahlen verzeichnen und ein sehr homogenes Angebot sicherstellen kann. Dieses zentrale OAR ist eingebettet in die eher dezentral politisch wirkende Organisation namens Archives Ouvertes. 
Auch die nordischen Länder warten mit hoch entwickelten Netzwerken auf, auf technischer und organisatorischer Basis: Die „Danish Research Database“ in Dänemark basiert auf IRs, in Schweden gibt es technische Netzwerke (DIVA, s. o.) nationale Netzwerke ${ }^{17}$ und international renommierte Aktivitäten, wie etwa an der Universitätsbibliothek Lund ${ }^{18}$. In Finnland existieren integrierte nationale Strukturen $^{19}$, in Norwegen eine Mischung aus technischen und organisatorischen Aktivitäten ${ }^{20}$. Starke Initiativen finden sich auch Irland ${ }^{21}$, Litauen $^{22}$, Portugal ${ }^{23}$, Slovenien ${ }^{24}$ und Spanien ${ }^{25}$. Ein besonders interessantes Beispiel für nationale Vernetzung in jüngster Zeit bietet Belgien, bei dem - stimuliert durch das Europäische Projekt DRIVER (s. u.) - 13 Rektoren sowohl aus dem flämischen als auch aus dem wallonischen Teil Belgiens - auf einer gemeinsamen Veranstaltung die Berliner Erklärung ${ }^{26}$ unterschrie- $^{-}$ ben und damit eine einheitliche politische Basis für die praktische Vernetzung ihrer IRs gelegt haben.

\section{Europa}

Europa bietet mit all diesen nationalen Initiativen wohl die weltweit vielfältigste und aktivste Landschaft für IRs und hat damit alle Voraussetzungen, eine führende Rolle bei der Etablierung eines infrastrukturell verankerten Netzwerkes von IRs einzunehmen. Keiner bezweifelt zudem, dass die internationale Vernetzung bei der zunehmenden Globalisierung der Wissenschaft eine unabdingbare Notwendigkeit ist. Denn auch die nationalen Bestände sind für viele Wissenschaften keine ausreichende Basis für ihre Recherchen. Um das Potenzial in Europa als Zwischenschritt für die globale ErschlieBung auszuschöpfen, ist es also notwendig, dass auch auf dieser kontinentalen Ebene die Vernetzung vorangetrieben wird. Das Projekt DRIVER ${ }^{27}$ - „Digital Repository Vision for European Research” hat sich genau dieser Aufgabe verschrieben. Neben technischen Aktivitäten (s. u.) ist das organisatorische Ziel die Vernetzung bestehender nationaler Initiativen und die Stimulierung der Entwicklung in Ländern ohne derartige Strukturen, wie in dem o. g. Beispiel aus Belgien. In der derzeit laufenden Projektphase wird eine Testumgebung mit Belgien, Deutschland, Frankreich, Großbritannien und den Niederlanden unter Einbeziehung der entsprechenden Organisationen DINI, HAL bzw. Archives Ouvertes, SHERPA und DARE betrieben. Die hierin gewonnenen Erkenntnisse sollen beim Aufbau einer organisatorischen Struktur helfen, die in weiteren Schritten alle anderen europäischen Länder berücksichtigt.

Horstmann: Open Access international - lokale Systeme, kooperative Netzwerke und S. 232 visionäre Infrastrukturen. ZfBB 54 (2007) 4-5, S.230-233.

\section{Die globale Herausforderung}

Die offensichtliche globale Herausforderung liegt darin, alle weltweit verteilten Inhalte systematisch zugänglich zu machen. Hierzu müssen die Katalogeinträge (Metadaten) der IRs ,,aggregiert“ werden um Wissenschaftlern mit Hilfe von Suchmaschinen, die viele IRs indexieren, einen umfassenden Zugriff auf lokal verteilte Inhalte zu ermöglichen. Genau hier liegt eine Stärke des ,Paradigmas' von IRs, die es möglich macht, dass sie das Rückgrat der OA-Aktivitäten bilden. Es ist über einen technischen Standard für lokale IRs, dem Open-Archives-Initiative-Protocol for Metadata-Harvesting ${ }^{28}$ (OAI-PMH) konzeptionell, organisatorisch und technisch möglich, relativ einfach Homogenität in der Vernetzung von IRs herbeizuführen, indem Daten lokaler IRs fortwährend automatisch „,geerntet“ (engl. harvesting) werden. OAIster ${ }^{29}$ ist ein Beispiel für eine Suchmaschine aus den USA, die einen solchen globalen Zugang herstellt. Die Bielefeld Academic Search Engine ${ }^{30}$ (BASE) ist der deutsche Beitrag zu dieser Form von Dienstleistung. BASE wird international verwendet und sogar von der Open Archive Initiative empfohlen. Google Scholar bietet ebenfalls Zugriff auf Inhalte aus IRs, ist jedoch nicht darauf beschränkt, so dass Suchergebnisse in Google Scholar ${ }^{31}$ nicht selten auf lizenzpflichtige Dokumente verweisen.

Nun kann man kann sich natürlich die Frage stellen: „Warum brauchen wir eigentlich regionale oder nationale Netzwerke, wenn es doch einen globalen Zugang gibt, der direkt auf die lokalen IRs zugreift?" Der Grund ist bei einer genaueren Betrachtung der Suchergebnisse aus globalen Suchmaschinen sofort offensichtlich. Die Inhalte, die in lokalen IRs angeboten werden, sind sehr heterogen und wenig standardisiert. Dies gilt sowohl für Inhalte innerhalb eines IRs, Inhalte in verschiedenen IRs eines Landes, in verschiedenen Ländern, oder zu verschiedenen Themen. Die Suchergebnisse sind dementsprechend wenig spezifisch. Suchmaschinen wie BASE und OAIster investieren zwar viel in 
die intelligente Strukturierung der Daten, die beispielsweise eine themenspezifische Suche möglich macht, aber alle Unterschiede lokaler Konventionen, können so nicht herausgefiltert werden. Darüber hinaus ist klar: Daten, die lokal nicht vorhanden sind, können schwerlich zentral zur Verfügung gestellt werden. Netzwerke werden also u. a. benötigt, um Standards zu etablieren und den Ausbau und die Verbesserung von IRs lokal, regional, national oder kontinental voranzutreiben.

\section{Visionäre Infrastrukturen}

Es wird deutlich, dass die einfache Suche nicht das Ende einer IR-Infrastruktur ist - tatsächlich sind Suchmaschinen nur der Anfang. Die zugrunde liegende Vision ist vielmehr, dass auf Basis von OAInhalten und den nationalen oder internationalen Netzwerken eine neue Form von wissenschaftlichem Informationsmanagement aufgebaut werden kann, wie es etwa das Beispiel DAREnet in den Niederlanden in Ansätzen zeigt (s. o.). Mehrwertfunktionen, wie offene und transparente Zitationsanaly$\operatorname{sen}^{32}$, Annotationen oder vereinfachte Nachnutzung der Inhalte werden zum Teil bereits heute durch spezialisierte Internetdienstleistungen zur Verfügung gestellt. Wissenschaftliche Artikel werden als ,Publikationspaket' gemeinsam mit Zusatzmaterialien, etwa Rohdaten oder elektronischen Tutorien zur Verfügung gestellt, um eine bessere wissenschaftliche Praxis zu ermöglichen. IRs werden innerhalb einer Institution an die Programme zur Forschungsverwaltung gekoppelt ${ }^{33}$ und können beispielsweise mit E-Learning-Systemen zusammenarbeiten.

Deutschland trägt dazu bei, dass auch diese Visionen Realität werden. Entwicklungsprojekte im Kontext von DINI e.V., eingebettet im internationalen Kontext, werden kontinuierlich durchgeführt. DINI e.V. ist ferner aktiv in Knowledge Exchange ${ }^{34}$, einer gemeinsamen Initiative der Deutschen Forschungsgemeinschaft (DFG), des Joint Research Information Committee ${ }^{35}$ (JISC) in Großbritannien, $\mathrm{SURF}^{36}$ in den Niederlanden und $\mathrm{DEFF}^{37}$ in Dänemark. Hier werden die nächsten Schritte der Entwicklung von IRs thematisiert, beispielsweise die Kopplung an Autorisierungssysteme, die verhindern, dass Wissenschaftler sich in verschiedenen Diensten der Universität, das Landes oder in Europa jeweils stets erneut anmelden müssen. In Europa kümmert sich DRIVER neben den oben genannten organisatorischen Aufgaben um die die Bereitstellung eines harmonisierten Europäischen Datenraumes, auf den Mehrwertdienste zugreifen können ohne andernfalls notwendige Datenverarbeitung erneut vornehmen zu müssen. DRIVER stellt auch eine Infrastruktur zur Verfügung, in der die Landschaft von IRs zusammengefasst und verschiedenste Mehrwertdienste orchestriert werden.

Die Welt der IRs relativ einfach, da sie zunächst nur einen sehr kleinen Ausschnitt des Lebenszyklus wissenschaftlicher Information bedient: IRs stellen lokal Daten für die weltweite Nachnutzung zur Verfügung. OA-Zeitschriften (der „Goldenen Weg“) hingegen bilden große Teile dieses Lebenszyklus ab. Die vergleichsweise einfache Welt von IRs wird jedoch vor dem Hintergrund einer weltweiten Vernetzung und einer kooperativen Dateninfrastruktur zunehmend reichhaltiger. Sie nähert sich der Welt der OA-Zeitschriften an: Ist die Grundlage einer umfassenden Dateninfrastruktur und im Internet harmonisie-

$$
\begin{gathered}
\text { Horstmann: Open Access international - lokale Systeme, kooperative Netzwerke und } \quad \text { S. } 233 \\
\text { visionäre Infrastrukturen. ZfBB } 54 \text { (2007) 4-5, S.230-233. }
\end{gathered}
$$

renden Zusatzfunktionen erst einmal vorhanden, ist der Schritt zu ausgereiften internetbasierten Publikationssystemen mit Editierwerkzeugen und Begutachtungsfunktionen in virtuellen Fachgemeinschaften nicht mehr weit.

\footnotetext{
${ }^{1}$ Der Begriff der offenen Digitalen Archive (Open Access Repositories, OAR) wird hier als Sammelbegriff für institutionell verankerte Archive (engl. ,institutional repositories“) und disziplinäre Archive (engl. „subjectbased repositories") verwendet.

${ }^{2} \mathrm{http}$ ///www.eprints.org/openaccess/

${ }^{3}$ Im Gegenteil, nur die Entwicklungen im Elektronischen Publizieren können einen langfristigen Erfolg von OA garantieren.

${ }^{4}$ http://arxiv.org/

${ }^{5} \mathrm{http}: / /$ www.pubmedcentral.nih.gov/
} 


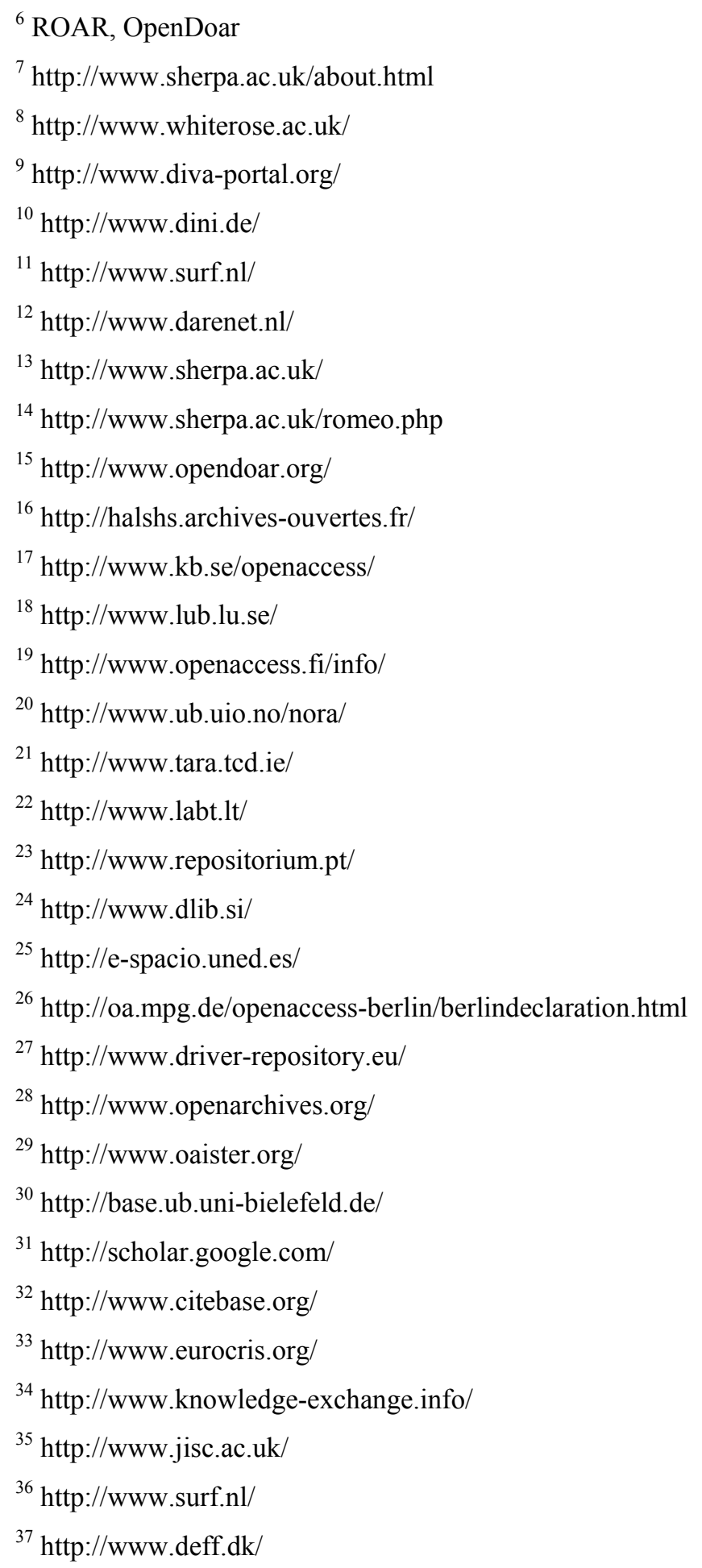

